\title{
Fuzzy semi-numbers and a distance on them with a case study in medicine
}

\author{
Sh. Yeganehmanesh ${ }^{1} \cdot$ M. Amirfakhrian ${ }^{1}$ (D) P. Grzegorzewski ${ }^{2,3}$
}

Received: 2 September 2017 / Accepted: 7 January 2018/ Published online: 22 January 2018

(C) The Author(s) 2018, corrected publication February 2018. This article is an open access publication

\begin{abstract}
In this paper, we present the novel concept of fuzzy semi-numbers. Then, a method for assigning distance between every pair of fuzzy semi-numbers is given. Moreover, it is shown that this distance is a metric on the set of all trapezoidal fuzzy semi-numbers with the same height and is a pseudo-metric on the set of all fuzzy semi-numbers. Also, by utilizing this distance, we propose an approximation of a fuzzy semi-number with given height and apply this approximation method in a medical case study.
\end{abstract}

Keywords Fuzzy sets · Fuzzy numbers · Fuzzy semi-numbers · Generalized LR fuzzy semi-numbers $\cdot H$-value $\cdot$ $H$-ambiguity

\section{Introduction}

Conventional works on fuzzy logic assume that fuzzy sets satisfy the conditions of convexity and normality (the height is one) which are called fuzzy numbers. Many of the researches in fuzzy logic have contributed to the approximation methods of a fuzzy number. Such an approximation has been done in several ways. Some authors have assigned a crisp number to a fuzzy number as a ranking method. Such methods suffer from a great amount of data loss. Some other methods $[10,14]$ have defined an interval as an approximation of a fuzzy number. But, in this case, the modal value (the core with height 1) of the fuzzy numbers is lost. In some works such as $[1,3,13,15,16]$, the authors have tried to solve

The original version of this article was revised: The spelling of the third author's name was incorrect.

M. Amirfakhrian

amirfakhrian@iauctb.ac.ir

1 Department of Mathematics, Central Tehran Branch, Islamic Azad University, Tehran, Iran

2 Systems Research Institute, Polish Academy of Sciences, Newelska 6, 01-447 Warsaw, Poland

3 Faculty of Mathematics and Information Science, Warsaw University of Technology, Plac Politechniki 1, 00-661 Warsaw, Poland an optimization problem to obtain a trapezoidal fuzzy number as a nearest approximation. Recently, some works have been done on approximation of a fuzzy number $[4,6-9,11]$ by defining distance functions.

All the aforementioned methods destroy much of the useful data associated with the fuzzy number and as a result imprecision of the approximation increases. Such loss is not negligible specially in high critical domains such as medicine where most of the clinical data are intrinsically inexact and ambiguous. To mitigate this, most often applying fuzzy mathematical modeling is favorable approach. However, these data are not necessarily normal fuzzy sets. To overcome these problems, this paper works on fuzzy sets in general form and without regard to their height. At first, we present the novel concept of fuzzy seminumber. Then, we propose a distance to approximate an arbitrary fuzzy semi-number. Depending on some predefined conditions, the result of approximation can be either a fuzzy number or fuzzy semi-number. To this end, in this paper an approximation for a given fuzzy set without considering their height is proposed. Also, for more clarification, a real application of fuzzy semi-number will be studied in terms of a case study.

The structure of the this paper is as follows. In "Preliminaries", the basic concepts of our work are introduced; then in next section, we review fuzzy semi-numbers. Section "Height source distance between fuzzy semi-numbers" introduces a new distance namely Height Source 
Distance for fuzzy semi-numbers. In next section, the nearest trapezoidal fuzzy semi-number to an arbitrary fuzzy semi-number is introduced and a simple method for its computation is presented. Next section contains some numerical examples. Section "A medical case study" presents a case study in medicine and the final section concludes the article.

\section{Preliminaries}

Before delving in to our contributions, let us take a glance at some basic fuzzy theory definitions. Let $F(\mathbb{R})$ be the set of all real fuzzy numbers (which are normal, upper semicontinuous, convex and compactly supported fuzzy sets).

The parametric form of a fuzzy number is denoted by $\tilde{u}=(\underline{u}, \bar{u})$, where functions $\underline{u}$ and $\bar{u}$ for each $\alpha \in[0,1]$ satisfy the following requirements $[18,19]$ :

1. $\underline{u}$ is a monotonically increasing left continuous function.

2. $\bar{u}$ is a monotonically decreasing left continuous function.

3. $\underline{u}(\alpha) \leq \bar{u}(\alpha), \quad 0 \leq \alpha \leq 1$.

Let $\tilde{u}=(\underline{u}, \bar{u}), \tilde{v}=(\underline{v}, \bar{v}) \in F(\mathbb{R})$. Some results of applying fuzzy arithmetic on fuzzy numbers $\tilde{u}$ and $\tilde{v}$ are as follows:

- $x>0: x \tilde{u}=(x \underline{u}, x \bar{u})$;

- $x<0: x \tilde{u}=(x \bar{u}, x \underline{u})$

- $\tilde{u}+\tilde{v}=(\underline{u}+\underline{v}, \bar{u}+\bar{v})$;

- $\tilde{u}-\tilde{v}=(\underline{u}-\bar{v}, \bar{u}-\underline{v})$.

A fuzzy number $\tilde{v}$ is non-negative (non-positive) if for $x<0(x>0)$, we have $\mu_{\tilde{v}}(x)=0$, equivalently if on $[0,1]$ we have $\underline{v} \geq 0(\bar{v} \leq 0)$. Also a fuzzy number $\tilde{v}$ is positive (negative) if for $x \leq 0(x \geq 0)$, we have $\mu_{\tilde{v}}(x)=0$, equivalently if on $[0,1]$ we have $\underline{v}>0(\bar{v}<0)$. For $\alpha \in(0,1], \alpha-$ cut of a fuzzy set $\tilde{w}$ is defined as $[\tilde{w}]^{\alpha}=\left\{x \mid \mu_{\tilde{w}}(x) \geq \alpha\right\}$ [23].

Definition 1 A function $s \in C[0,1]$ with the following properties is a source function [2,5] (regular reducing function in [20]) over all fuzzy numbers:

1. $s(\alpha) \geq 0, \quad \alpha \in[0,1]$

2. $s(0)=0$,

3. $s(1)=1$,

4. $\int_{0}^{1} s(\alpha) \mathrm{d} \alpha=\frac{1}{2}$.

Definition 2 The core of a fuzzy number $\tilde{u}$ is defined as follows:

$$
\text { core }(\tilde{u})=\left\{x \mid \mu_{\tilde{u}}(x)=1\right\}
$$

Definition 3 Let $s$ be a source function defined on $F(\mathbb{R})$. For $\tilde{u} \in F(\mathbb{R})$, value and ambiguity associated with $s$ are defined by the following relations [20]:

1. $\mathrm{V}(\tilde{u})=\int_{0}^{1} s(\alpha)[\bar{u}(\alpha)+\underline{u}(\alpha)] \mathrm{d} \alpha$,

2. $\mathrm{A}(\tilde{u})=\int_{0}^{1} s(\alpha)[\bar{u}(\alpha)-\underline{u}(\alpha)] \mathrm{d} \alpha$.

\section{Fuzzy semi-numbers}

Here, we present several novel definitions and lemmas which will be used throughout the paper.

Definition 4 A fuzzy set $\tilde{u}$ (with the membership function $\mu_{\tilde{u}}$ ) is a generalized fuzzy LR semi-number, if there exist a positive number $h \in(0,1]$ such that

$\mu_{\tilde{u}}(x)=\left\{\begin{array}{cc}l(x), & a \leq x \leq b, \\ h, & b \leq x \leq c, \\ r(x), & c \leq x \leq d, \\ 0, & \text { otherwise }\end{array}\right.$

where $l(x)$ is nondecreasing on $[a, b]$ and $r(x)$ is nonincreasing on $[c, d]$ such that $l(a)=r(d)=0$ and $l(b)=$ $r(c)=h$ and we denote fuzzy LR semi-number by $(a, b, c, d ; h)_{\mathrm{LR}}$. We show fuzzy semi-number $\tilde{u}$ with height $h$ as $\tilde{u}_{h}$ and if $h=1$ then $\tilde{u}$ is an LR fuzzy number [23]. We denote the set of all fuzzy numbers by $F(\mathbb{R})$ and the set of all fuzzy semi-numbers of height $h$ by $F_{h}(\mathbb{R})$. Also the set of all fuzzy semi-numbers is denoted by $\mathrm{FS}(\mathbb{R})$. i.e.,

$\mathrm{FS}(\mathbb{R}):=\bigcup_{h \in(0,1]} F_{h}(\mathbb{R})$,

it is clear that $F(\mathbb{R})$ is a proper subset of $\mathrm{FS}(\mathbb{R})$. (Actually, $F(\mathbb{R})$ includes the fuzzy sets with height one, however, $\mathrm{FS}(\mathbb{R})$ includes fuzzy sets with arbitrary heights.)

A fuzzy semi-number $\tilde{v}_{h}$ is non-negative (non-positive) if for $x<0(x>0)$, we have $\mu_{\tilde{v}}(x)=0$, equivalently if on $[0, h]$ we have $\underline{v} \geq 0(\bar{v} \leq 0)$. Also a fuzzy semi-number $\tilde{v}_{h}$ is positive (negative) if for $x \leq 0(x \geq 0)$, we have $\mu_{\tilde{v}}(x)=0$, equivalently if on $[0, h]$ we have $\underline{v}>0(\bar{v}<0)$.

In addition, if $l(x)$ and $r(x)$ are linear, then $\tilde{u}_{h}$ is a trapezoidal fuzzy semi-number which is denoted by $(a, b, c, d ; h)$. In this case if $b=c$, we denote it by $(a, b, d ; h)$, which is a triangular fuzzy semi-number. Furthermore, let $T F(\mathbb{R})$ and $T F_{h}(\mathbb{R})$ be the set of all trapezoidal fuzzy numbers and all trapezoidal fuzzy seminumbers on $\mathbb{R}$ with height $h$, respectively:

$\left\{\begin{array}{l}T F(\mathbb{R})=\{(a, b, c, d): a \leq b \leq c \leq d\}, \\ T F_{h}(\mathbb{R})=\{(a, b, c, d ; h): a \leq b \leq c \leq d, 0<h \leq 1\},\end{array}\right.$ 
we denote the set of all trapezoidal fuzzy semi-numbers with arbitrary heights by $\operatorname{TFS}(\mathbb{R})$

$\operatorname{TFS}(\mathbb{R}):=\bigcup_{h \in(0,1]} T F_{h}(\mathbb{R})$.

Definition 5 The core of a fuzzy semi-number $\tilde{u}_{h}$ with height $h \in(0,1]$ is called H-core and is defined as follows:

$$
\text { H-core }\left(\tilde{u}_{h}\right)=\left\{x \mid \mu_{\tilde{u}}(x)=h\right\}
$$

Definition 6 A function $s_{h} \in C[0, h]$ with the following properties is a source function:

1. $s_{h}(\alpha) \geq 0, \quad \alpha \in[0, h]$

2. $s_{h}(0)=0$,

3. $s_{h}(h)=h$,

4. $\int_{0}^{h} s_{h}(\alpha) \mathrm{d} \alpha=\frac{1}{2} h^{2}$.

Definition 7 Let $s_{h_{1}}$ be a source function over $\left[0, h_{1}\right] . s_{h_{2}}$ is equivalent to $s_{h_{1}}$ if for a given height $h_{2}, s_{h_{2}}$ is defined as $s_{h_{2}}(\alpha)=\frac{h_{2}}{h_{1}} s_{h_{1}}\left(\frac{h_{1}}{h_{2}} \alpha\right)$. This equivalence is denoted by $s_{h_{1}} \approx s_{h_{2}}$

Lemma 1 Let $s_{h_{1}}$ be a source function over $\left[0, h_{1}\right]$. If $s_{h_{2}} \approx s_{h_{1}}$, then $s_{h_{2}}$ is a source function over $\left[0, h_{2}\right]$.

Proof For $s_{h_{2}}$ we have the following relations:

1. $s_{h_{2}}(\alpha)=\frac{h_{2}}{h_{1}} s_{h_{1}}\left(\frac{h_{1}}{h_{2}} \alpha\right) \geq 0, \quad \alpha \in\left[0, h_{2}\right]$

2. $s_{h_{2}}(0)=\frac{h_{2}}{h_{1}} s_{h_{1}}(0)=0$,

3. $s_{h_{2}}\left(h_{2}\right)=\frac{h_{2}}{h_{1}} s_{h_{1}}\left(\frac{h_{1}}{h_{2}} h_{2}\right)=h_{2}$,

4. $\int_{0}^{h_{2}} s_{h_{2}}(\alpha) \mathrm{d} \alpha=\frac{h_{2}}{h_{1}} \int_{0}^{h_{2}} s_{h_{1}}\left(\frac{h_{1}}{h_{2}} \alpha\right) \mathrm{d} \alpha=$ $\frac{h_{2}}{h_{1}} \frac{h_{2}}{h_{1}} \int_{0}^{h_{1}} s_{h_{1}}(\beta) \mathrm{d} \beta=\frac{1}{2} h_{2}^{2}$,

where $\beta=\frac{h_{1}}{h_{2}} \alpha$ and $\mathrm{d} \beta=\frac{h_{1}}{h_{2}} \mathrm{~d} \alpha$.

Hence $s_{h_{2}}$ is a source function over $\left[0, h_{2}\right]$.

For a fuzzy semi-number $\tilde{u}_{h} \in F_{h}(\mathbb{R})$ and $\alpha \in[0, h]$ we define $\underline{u}(\alpha)$ and $\bar{u}(\alpha)$ as follows:

$\underline{u}(\alpha)= \begin{cases}\inf \left\{x: \mu_{\tilde{u}_{h}}(x) \geq \alpha\right\}, & \alpha \in(0, h], \\ \inf \operatorname{supp}\left(\mu_{\tilde{u}_{h}}(x)\right), & \alpha=0 .\end{cases}$
$\bar{u}(\alpha)= \begin{cases}\sup \left\{x: \mu_{\tilde{u}_{h}}(x) \geq \alpha\right\}, & \alpha \in(0, h], \\ \sup \operatorname{supp}\left(\mu_{\tilde{u}_{h}}(x)\right), & \alpha=0 .\end{cases}$

So the parametric form of a fuzzy semi-number is denoted by $\tilde{u}_{h}=(\underline{u}, \bar{u} ; h)$, where functions $\underline{u}$ and $\bar{u}$ satisfy the following requirements:

1. $\underline{u}$ is a monotonically increasing left continuous function over $[0, h]$.

2. $\bar{u}$ is a monotonically decreasing left continuous function over $[0, h]$.

3. $\underline{u}(\alpha) \leq \bar{u}(\alpha), \quad 0 \leq \alpha \leq h$.

4. $\underline{u}(\alpha)=\bar{u}(\alpha)=0$, for $\alpha \notin[0, h]$.
For a trapezoidal fuzzy semi-number which is denoted by $\tilde{u}=(a, b, c, d ; h)$, we have

$\underline{u}(\alpha)=a+\frac{b-a}{h} \alpha$,

$\bar{u}(\alpha)=d-\frac{d-c}{h} \alpha$.

Definition 8 Crisp semi-number $a_{h}^{\circ}$, is a fuzzy singleton set with height $h$, then $\underline{u}(\alpha)=\bar{u}(\alpha)=a$, for $\forall \alpha \in[0, h]$ and we denote $a_{h}^{\circ}=(a ; h)$. We denote the set of all crisp seminumbers of height $h$ by $\mathbb{R}_{h}^{\circ}$ and we have $\mathbb{R}_{1}^{\circ}=\mathbb{R}$. The additive identity on $F_{h}(\mathbb{R})$ is $0_{h}^{\circ}=(0 ; h)$.

Definition 9 Let $s_{h}$ be a source function defined over $[0, h]$. For a fuzzy semi-number $\tilde{u}_{h} \in F_{h}(\mathbb{R}), H$-value and $H$-ambiguity associated with $s_{h}$ are defined by the following relations:

1. $\quad \mathrm{HV}\left(\tilde{u}_{h}\right)=\int_{0}^{h} s_{h}(\alpha)[\bar{u}(\alpha)+\underline{u}(\alpha)] \mathrm{d} \alpha$,

2. $\quad \mathrm{HA}\left(\tilde{u}_{h}\right)=\int_{0}^{h} s_{h}(\alpha)[\bar{u}(\alpha)-\underline{u}(\alpha)] \mathrm{d} \alpha$.

Lemma 2 For $\tilde{u}_{h} \in F_{h}(\mathbb{R})$ and $k \in \mathbb{R}$, we have following properties:

1. $\mathrm{HV}\left(k \tilde{u}_{h}\right)=k \operatorname{HV}\left(\tilde{u}_{h}\right)$,

2. $\operatorname{HA}\left(k \tilde{u}_{h}\right)=|k| \operatorname{HA}\left(\tilde{u}_{h}\right)$.

Proof straightforward.

Lemma 3 For $\tilde{u}_{h} \in F_{h}(\mathbb{R})$ and $k \in \mathbb{R}$, we have following properties:

1. $\mathrm{HV}\left(\tilde{u}_{h}+k\right)=\operatorname{HV}\left(\tilde{u}_{h}\right)+h^{2} k$,

2. $\mathrm{HA}\left(\tilde{u}_{h}+k\right)=\mathrm{HA}\left(\tilde{u}_{h}\right)$.

Proof By definition of $H$-value and $H$-ambiguity we can write as follows:

1. $\mathrm{HV}\left(\tilde{u}_{h}+k\right)=\int_{0}^{h} s_{h}(\alpha)[\bar{u}(\alpha)+k+\underline{u}(\alpha)+k] \mathrm{d} \alpha$

$$
\begin{aligned}
& =\int_{0}^{h} s_{h}(\alpha)[\bar{u}(\alpha)+\underline{u}(\alpha)+2 k] \mathrm{d} \alpha \\
& =\int_{0}^{h} s_{h}(\alpha)[\bar{u}(\alpha)+\underline{u}(\alpha)] \mathrm{d} \alpha+2 k \int_{0}^{h} s_{h}(\alpha) \mathrm{d} \alpha \\
& =\operatorname{HV}\left(\tilde{u}_{h}\right)+h^{2} k .
\end{aligned}
$$

2. $\mathrm{HA}\left(\tilde{u}_{h}+k\right)=\int_{0}^{h} s_{h}(\alpha)[\bar{u}(\alpha)+k-(\underline{u}(\alpha)+k)] \mathrm{d} \alpha$

$$
\begin{aligned}
& =\int_{0}^{h} s_{h}(\alpha)[\bar{u}(\alpha)-\underline{u}(\alpha)] \mathrm{d} \alpha \\
& =\operatorname{HA}\left(\tilde{u}_{h}\right) .
\end{aligned}
$$

Lemma 4 In $\mathrm{FS}(\mathbb{R})$, for $H$-value and $H$-ambiguity we have following properties:

1. $\operatorname{HV}\left(\tilde{u}_{h} \pm \tilde{v}_{h}\right)=\operatorname{HV}\left(\tilde{u}_{h}\right) \pm \operatorname{HV}\left(\tilde{v}_{h}\right)$

2. $\mathrm{HA}\left(\tilde{u}_{h} \pm \tilde{v}_{h}\right)=\operatorname{HA}\left(\tilde{u}_{h}\right)+\operatorname{HA}\left(\tilde{v}_{h}\right)$. 
Proof straightforward.

Definition 10 Let $s$ be a source function, then the source number $I_{s, h}$ is defined on $s$ as follows:

$I_{s, h}=\int_{0}^{h} s(\alpha) \alpha \mathrm{d} \alpha$

Lemma 5 If $s_{1}$ and $s_{2}$ are equivalent source functions defined over $\left[0, h_{1}\right]$ and $\left[0, h_{2}\right]$, respectively, then we have:

$I_{s_{2}, h_{2}}=\left(\frac{h_{2}}{h_{1}}\right)^{3} I_{s_{1}, h_{1}}$

Proof Using Definition 10 we have:

$$
\begin{aligned}
I_{s_{2}, h_{2}} & =\int_{0}^{h_{2}} s_{h_{2}}(\alpha) \alpha \mathrm{d} \alpha=\frac{h_{2}}{h_{1}} \int_{0}^{h_{2}} s_{h_{1}}\left(\frac{h_{1}}{h_{2}} \alpha\right) \alpha \mathrm{d} \alpha \\
& =\left(\frac{h_{2}}{h_{1}}\right)^{2} \int_{0}^{h_{1}} s_{h_{1}}(\beta)\left(\frac{h_{2}}{h_{1}} \beta\right) d \beta \\
& =\left(\frac{h_{2}}{h_{1}}\right)^{3} \int_{0}^{h_{1}} s_{h_{1}}(\beta) \beta d \beta=\left(\frac{h_{2}}{h_{1}}\right)^{3} I_{s_{1}, h_{1}}
\end{aligned}
$$

where $\beta=\frac{h_{1}}{h_{2}} \alpha$ and $\mathrm{d} \beta=\frac{h_{1}}{h_{2}} \mathrm{~d} \alpha$.

Lemma 6 For an arbitrary fuzzy semi-number $\tilde{u}$ with height $h$, we have $I_{s, h}<\frac{1}{2} h^{3}$.

Proof By Mid-point Theorem, the proof is straightforward.

\section{Height source distance between fuzzy semi- numbers}

In this section, we introduce a new distance function namely Height Source Distance to measure the distance between fuzzy semi-numbers which is invariant on translation only in cases where heights are the same.

Definition 11 For $\tilde{u}_{h_{u}}, \tilde{v}_{h_{v}} \in \mathrm{FS}(\mathbb{R})$ with heights $h_{u}$ and $h_{v}$, respectively, Height Source Distance HSD, is defined as follows:

$$
\begin{aligned}
& \operatorname{HSD}\left(\tilde{u}_{h_{u}}, \tilde{v}_{h_{v}}\right) \\
& =\left\{\begin{array}{cc}
\frac{1}{2}\left\{\left|h_{u} \operatorname{HV}\left(\tilde{u}_{h_{u}}\right)-h_{v} \operatorname{HV}\left(\tilde{v}_{h_{v}}\right)\right|\right. & \text { if } \tilde{u} \neq 0_{h_{u}}^{\circ} \text { or } \tilde{v} \neq 0_{h_{v}}^{\circ} \\
+\left|h_{u} \operatorname{HA}\left(\tilde{u}_{h_{u}}\right)-h_{v} \operatorname{HA}\left(\tilde{v}_{h_{v}}\right)\right| & \text { if } \tilde{u}=0_{h_{u}}^{\circ} \text { and } \tilde{v}=0_{h_{v}}^{\circ} \\
+d_{H}\left(h_{u}^{2}\left[\tilde{u}^{h_{u}}, h_{v}^{2}[\tilde{v}]^{h_{v}}\right)\right\}, & h_{u}-h_{v} \mid,
\end{array}\right.
\end{aligned}
$$

where $d_{H}$ is the Hausdorff meter, and $[\tilde{w}]^{h}$ is the $h$-cut of fuzzy set $\tilde{w}$.

In the following, we present some theorems of the proposed distance function and investigate its properties from the analytic geometry perspective.
Theorem $7 \quad$ For $\tilde{u}_{h_{u}}, \tilde{v}_{h_{v}}, \tilde{w}_{h_{w}} \in \mathrm{FS}(\mathbb{R})$ and $k \in \mathbb{R}, H S D$ satisfies the following properties:

1. $\operatorname{HSD}\left(\tilde{u}_{h_{u}}, \tilde{u}_{h_{u}}\right)=0$,

2. $\operatorname{HSD}\left(\tilde{u}_{h_{u}}, \tilde{v}_{h_{v}}\right)=\operatorname{HSD}\left(\tilde{v}_{h_{v}}, \tilde{u}_{h_{u}}\right)$,

3. $\operatorname{HSD}\left(\tilde{u}_{h_{u}}, \tilde{w}_{h_{w}}\right) \leq \operatorname{HSD}\left(\tilde{u}_{h_{u}}, \tilde{v}_{h_{v}}\right)+\operatorname{HSD}\left(\tilde{v}_{h_{v}}, \tilde{w}_{h_{w}}\right)$,

4. $\operatorname{HSD}\left(k \tilde{u}_{h_{u}}, k \tilde{v}_{h_{v}}\right)=|k| \operatorname{HSD}\left(\tilde{u}_{h_{u}}, \tilde{v}_{h_{v}}\right)$.

Proof If $\tilde{u}=0_{h_{u}}^{\circ}$ and $\tilde{v}=0_{h_{v}}^{\circ}$ then the proof is trivial; otherwise, for each step we can write as follows:

1. Since $d_{H}$ is a meter we have:

$$
\begin{aligned}
\operatorname{HSD}\left(\tilde{u}_{h_{u}}, \tilde{u}_{h_{u}}\right)= & \frac{1}{2}\left\{\left|h_{u} \operatorname{HV}\left(\tilde{u}_{h_{u}}\right)-h_{u} \operatorname{HV}\left(\tilde{u}_{h_{u}}\right)\right|\right. \\
& +\left|h_{u} \operatorname{HA}\left(\tilde{u}_{h_{u}}\right)-h_{u} \operatorname{HA}\left(\tilde{u}_{h_{u}}\right)\right| \\
& \left.+d_{H}\left(h_{u}^{2}\left[\tilde{u}_{h_{u}}\right]^{h_{u}}, h_{u}^{2}\left[\tilde{u}_{h_{u}}\right]^{h_{u}}\right)\right\}=0
\end{aligned}
$$

2. Since $d_{H}$ is a meter we have:

$$
\begin{aligned}
\operatorname{HSD}\left(\tilde{u}_{h_{u}}, \tilde{u}_{h_{u}}\right)= & \frac{1}{2}\left\{\left|h_{u} \operatorname{HV}\left(\tilde{u}_{h_{u}}\right)-h_{u} \operatorname{HV}\left(\tilde{u}_{h_{u}}\right)\right|\right. \\
& +\left|h_{u} \operatorname{HA}\left(\tilde{u}_{h_{u}}\right)-h_{u} \operatorname{HA}\left(\tilde{u}_{h_{u}}\right)\right| \\
& \left.+d_{H}\left(h_{u}^{2}\left[\tilde{u}_{h_{u}}\right]^{h_{u}}, h_{u}^{2}\left[\tilde{u}_{h_{u}}\right]^{h_{u}}\right)\right\}=0
\end{aligned}
$$

3. By $d_{H}$ is a meter and triangle inequality we have:

$$
\begin{aligned}
& \operatorname{HSD}\left(\tilde{u}_{h_{u}}, \tilde{w}_{h_{w}}\right)=\frac{1}{2}\left\{\left|h_{u} \operatorname{HV}\left(\tilde{u}_{h_{u}}\right)-h_{w} \operatorname{HV}\left(\tilde{w}_{h_{w}}\right)\right|\right. \\
&\left.\quad+\left|h_{u} \operatorname{HA}\left(\tilde{u}_{h_{u}}\right)-h_{w} \operatorname{HA}\left(\tilde{w}_{h_{w}}\right)\right|+d_{H}\left(h_{u}^{2}\left[\tilde{u}_{h_{u}}\right]^{h_{u}}, h_{w}^{2}\left[\tilde{w}_{h_{w}}\right]^{h_{w}}\right)\right\} \\
&=\frac{1}{2}\left\{\left|h_{u} \operatorname{HV}\left(\tilde{u}_{h_{u}}\right)-h_{v} \operatorname{HV}\left(\tilde{v}_{h_{v}}\right)+h_{v} \operatorname{HV}\left(\tilde{v}_{h_{v}}\right)-h_{w} \operatorname{HV}\left(\tilde{w}_{h_{w}}\right)\right|\right. \\
&+\left|h_{u} \operatorname{HA}\left(\tilde{u}_{h_{u}}\right)-h_{v} \operatorname{HA}\left(\tilde{v}_{h_{v}}\right)+h_{v} \operatorname{HA}\left(\tilde{v}_{h_{v}}\right)-h_{w} \operatorname{HA}\left(\tilde{w}_{h_{w}}\right)\right| \\
&\left.+d_{H}\left(h_{u}^{2}\left[\tilde{u}_{h_{u}}\right]^{h_{u}}, h_{w}^{2}\left[\tilde{w}_{h_{w}}\right]^{h_{w}}\right)\right\} \\
& \leq \frac{1}{2}\left\{h_{u}\left|\operatorname{HV}\left(\tilde{u}_{h_{u}}\right)-h_{v} \operatorname{HV}\left(\tilde{v}_{h_{v}}\right)\right|+\left|h_{v} \operatorname{HV}\left(\tilde{v}_{h_{v}}\right)-h_{w} \operatorname{HV}\left(\tilde{w}_{h_{w}}\right)\right|\right. \\
&+\left|h_{u} \operatorname{HA}\left(\tilde{u}_{h_{u}}\right)-h_{v} \operatorname{HA}\left(\tilde{v}_{h_{v}}\right)\right|+\left|h_{v} \operatorname{HA}\left(\tilde{v}_{h_{v}}\right)-h_{w} \operatorname{HA}\left(\tilde{w}_{h_{w}}\right)\right| \\
&+d_{H}\left(h_{u}^{2}\left[\tilde{u}_{h_{u}}\right]^{h_{u}}, h_{v}^{2}\left[\tilde{v}_{h_{v}}\right]^{h_{v}}\right)+d_{H}\left(h_{v}^{2}\left[\tilde{v}_{h_{v}}\right]^{h_{v}}, h_{w}^{2}\left[\tilde{w}_{h_{w}} h^{h_{w}}\right)\right\} \\
&= \frac{1}{2}\left\{\left|h_{u} \operatorname{HV}\left(\tilde{u}_{h_{u}}\right)-h_{v} \operatorname{HV}\left(\tilde{v}_{h_{v}}\right)\right|+\left|h_{u} \operatorname{HA}\left(\tilde{u}_{h_{u}}\right)-h_{v} \operatorname{HA}\left(\tilde{v}_{h_{v}}\right)\right|\right. \\
&\left.+d_{H}\left(h_{u}^{2}\left[\tilde{u}_{h_{u}}\right]^{h_{u}}, h_{v}^{2}\left[\tilde{v}_{h_{v}}\right]^{h_{v}}\right)\right\}+\frac{1}{2}\left\{\left|h_{v} \operatorname{HV}\left(\tilde{v}_{h_{v}}\right)-h_{w} \operatorname{HV}\left(\tilde{w}_{h_{w}}\right)\right|\right. \\
&\left.+\left|h_{v} \operatorname{HA}\left(\tilde{v}_{h_{v}}\right)-h_{w} \operatorname{HA}\left(\tilde{w}_{h_{w}}\right)\right| d_{H}\left(h_{v}^{2}\left[\tilde{v}_{h_{v}}\right]^{h_{v}}, h_{w}^{2}\left[\tilde{w}_{h_{w}}\right]^{h_{w}}\right)\right\} \\
&= \operatorname{HSD}\left(\tilde{u}_{h_{u}}, \tilde{v}_{h_{v}}\right)+\operatorname{HSD}\left(\tilde{v}_{h_{v}}, \tilde{w}_{h_{w}}\right) .
\end{aligned}
$$


4. By $d_{H}$ is a meter and Lemma 2 we have:

$$
\begin{aligned}
\operatorname{HSD}\left(k \tilde{u}_{h_{u}}, k \tilde{v}_{h_{v}}\right)=\frac{1}{2}\left\{\left|h_{u} \operatorname{HV}\left(k \tilde{u}_{h_{u}}\right)-h_{v} \operatorname{HV}\left(k \tilde{v}_{h_{v}}\right)\right|\right. \\
\left.\quad+\left|h_{u} \operatorname{HA}\left(k \tilde{u}_{h_{u}}\right)-h_{v} \operatorname{HA}\left(k \tilde{v}_{h_{v}}\right)\right|+d_{H}\left(h_{u}^{2}\left[k \tilde{u}_{h_{u}}\right]^{h_{u}}, h_{v}^{2}\left[k \tilde{v}_{h_{v}}\right]^{h_{v}}\right)\right\} \\
=\frac{1}{2}\left\{\left|k h_{u} \operatorname{HV}\left(\tilde{u}_{h_{u}}\right)-k h_{v} \operatorname{HV}\left(\tilde{v}_{h_{v}}\right)\right|+\left|k h_{u} \operatorname{HA}\left(\tilde{u}_{h_{u}}\right)-k h_{v} \operatorname{HA}\left(\tilde{v}_{h_{v}}\right)\right|\right. \\
\left.\quad+d_{H}\left(h_{u}^{2}\left[k \tilde{u}_{h_{u}}\right]^{h_{u}}, h_{v}^{2}\left[k \tilde{v}_{h_{v}}\right]^{h_{v}}\right)\right\} \\
=\frac{1}{2}\left\{|k|\left|h_{u} \operatorname{HV}_{h_{u}}(\tilde{u})-h_{v} \operatorname{HV}_{h_{v}}(\tilde{v})\right|+|k|\left|h_{u} \operatorname{HA}_{h_{u}}(\tilde{u})-h_{v} \operatorname{HA}_{h_{v}}(\tilde{v})\right|\right. \\
\left.\quad+|k| d_{H}\left(h_{u}^{2}[\tilde{u}]^{h_{u}}, h_{v}^{2}[\tilde{v}]^{h_{v}}\right)\right\} \\
=\frac{1}{2}|k|\left\{\left|\operatorname{HV}\left(\tilde{u}_{h_{u}}\right)-\operatorname{HV}\left(\tilde{v}_{h_{v}}\right)\right|+\left|\operatorname{HA}\left(\tilde{u}_{h_{u}}\right)-\operatorname{HA}\left(\tilde{v}_{h_{v}}\right)\right|\right. \\
\left.\quad+d_{H}\left(h_{u}^{2}\left[\tilde{u}_{h_{u}}\right]^{h_{u}}, h_{v}^{2}\left[\tilde{v}_{h_{v}}\right]^{h_{v}}\right)\right\}=|k| \operatorname{HSD}\left(\tilde{u}_{h_{u}}, \tilde{v}_{h_{v}}\right)
\end{aligned}
$$

For $\tilde{u}_{h}, \tilde{v}_{h} \in F_{h}(\mathbb{R})$ with equal height $h$, HSD simplified as:

$$
\begin{aligned}
& \operatorname{HSD}\left(\tilde{u}_{h}, \tilde{v}_{h}\right)=\frac{1}{2}\left\{h\left|\operatorname{HV}\left(\tilde{u}_{h}\right)-\operatorname{HV}\left(\tilde{v}_{h}\right)\right|+h \mid \operatorname{HA}\left(\tilde{u}_{h}\right)\right. \\
& \left.-\operatorname{HA}\left(\tilde{v}_{h}\right) \mid+h^{2} d_{H}\left(\left[\tilde{u}_{h}\right]^{h},\left[\tilde{v}_{h}\right]^{h}\right)\right\}
\end{aligned}
$$

Remark 8 For $\tilde{u}, \tilde{v} \in F(\mathbb{R})$, HSD, is the same as the source distance defined in [2].

Example 9 Let

$$
\begin{gathered}
\mu_{\tilde{u}}(x)= \begin{cases}h, & x=a, \\
0, & \text { otherwise, }\end{cases} \\
\mu_{\tilde{v}}(x)= \begin{cases}h, & x=b, \\
0, & \text { otherwise. }\end{cases} \\
\operatorname{HSD}\left(\tilde{u}_{h}, \tilde{v}_{h}\right)=\frac{1}{2}\left(h^{3}|a-b|+|0-0|+h^{2}|a-b|\right) \\
=\frac{1}{2} h^{2}(h+1)|a-b| .
\end{gathered}
$$

In this case if $\tilde{u}$ and $\tilde{v}$ are two crisp real numbers, then

$\operatorname{HSD}(\tilde{u}, \tilde{v})=|a-b|$.

For the set of all fuzzy semi-numbers of the same height, we prove the following theorem.

Theorem 10 For $\tilde{u}_{h}, \tilde{v}_{h}, \tilde{u}_{h}^{\prime}, \tilde{v}_{h}^{\prime} \in T F_{h}(\mathbb{R})$ and $k \in \mathbb{R}$, for $a$ fixed height $h$ and non-negative real number $k$, HSD satisfies the following properties:

1. $\operatorname{HSD}\left(\tilde{u}_{h}+k, \tilde{v}_{h}+k\right)=\operatorname{HSD}\left(\tilde{u}_{h}, \tilde{v}_{h}\right)$,

2. $\operatorname{HSD}\left(\tilde{u}_{h}+\tilde{v}_{h}, \tilde{u}_{h}^{\prime}+\tilde{v}_{h}^{\prime}\right) \leq \operatorname{HSD}\left(\tilde{u}_{h}, \tilde{u}_{h}^{\prime}\right)+\operatorname{HSD}\left(\tilde{v}_{h}, \tilde{v}_{h}^{\prime}\right)$.
Proof straightforward. The proof is obvious if the both $\tilde{u}=0_{h_{u}}^{\circ}$ and $\tilde{v}=0_{h_{v}}^{\circ}$ are satisfied.Thus, we investigate the proof in other cases. For the first part by $d_{H}$ is a meter and Lemma 3 we have:

$$
\begin{aligned}
& \operatorname{HSD}\left(\tilde{u}_{h}+k, \tilde{v}_{h}+k\right)=\frac{1}{2}\left\{\left|h \mathrm{HV}\left(\tilde{u}_{h}+k\right)-h \mathrm{HV}\left(\tilde{v}_{h}+k\right)\right|\right. \\
& \quad+\left|h \mathrm{HA}\left(\tilde{u}_{h}+k\right)-h \mathrm{HA}\left(\tilde{v}_{h}+k\right)\right| \\
& \left.+d_{H}\left(h^{2}\left[\tilde{u}_{h}+k\right]^{h}, h^{2}\left[\tilde{v}_{h}+k\right]^{h}\right)\right\} \\
& =\frac{1}{2}\left\{\left|h \mathrm{HV}\left(\tilde{u}_{h}\right)+h^{3} k-h \mathrm{HV}\left(\tilde{v}_{h}\right)-h^{3} k\right|\right. \\
& \quad+\left|h \mathrm{HA}\left(\tilde{u}_{h}\right)-h \mathrm{HA}\left(\tilde{v}_{h}\right)\right| \\
& \left.\quad+d_{H}\left(h^{2}\left[\tilde{u}_{h}\right]^{h}, h^{2}\left[\tilde{v}_{h}\right]^{h}\right)\right\} \\
& =\operatorname{HSD}\left(\tilde{u}_{h}, \tilde{v}_{h}\right)
\end{aligned}
$$

For the second part since $d_{H}$ is a meter by the triangle inequality and Lemma 4 we have:

$$
\begin{aligned}
& \operatorname{HSD}\left(\tilde{u}_{h}+\tilde{v}_{h}, \tilde{u}_{h}^{\prime}+\tilde{v}_{h}^{\prime}\right)=\frac{1}{2}\left\{\left|h \mathrm{HV}\left(\tilde{u}_{h}+\tilde{v}_{h}\right)-h \mathrm{HV}\left(\tilde{u}_{h}^{\prime}+\tilde{v}_{h}^{\prime}\right)\right|\right. \\
&+\left|h \mathrm{HA}\left(\tilde{u}_{h}+\tilde{v}_{h}\right)-h \mathrm{HA}\left(\tilde{u}_{h}^{\prime}+\tilde{v}_{h}^{\prime}\right)\right| \\
&\left.+d_{H}\left(h^{2}\left[\tilde{u}_{h}+\tilde{v}_{h}\right]^{h}, h^{2}\left[\tilde{u}_{h}^{\prime}+\tilde{v}_{h}^{\prime}\right]^{h}\right)\right\}, \\
&=\frac{1}{2}\left\{\left|h \mathrm{HV}\left(\tilde{u}_{h}\right)+h \mathrm{HV}\left(\tilde{v}_{h}\right)-h \mathrm{HV}\left(\tilde{u}_{h}^{\prime}\right)-h \mathrm{HV}\left(\tilde{v}_{h}^{\prime}\right)\right|\right. \\
&+\left|h \mathrm{HA}\left(\tilde{u}_{h}\right)+h \mathrm{HA}\left(\tilde{v}_{h}\right)-h \mathrm{HA}\left(\tilde{u}_{h}^{\prime}\right)-h \mathrm{HA}\left(\tilde{v}_{h}^{\prime}\right)\right| \\
&\left.+d_{H}\left(h^{2}\left[\tilde{u}_{h}+\tilde{v}_{h}\right]^{h}, h^{2}\left[\tilde{u}_{h}^{\prime}+\tilde{v}_{h}^{\prime}\right]^{h}\right)\right\}, \\
& \leq \frac{1}{2}\left\{\left|h \mathrm{HV}\left(\tilde{u}_{h}\right)-h \mathrm{HV}\left(\tilde{u}_{h}^{\prime}\right)\right|+\left|h \mathrm{HV}\left(\tilde{v}_{h}\right)-h \mathrm{HV}\left(\tilde{v}_{h}^{\prime}\right)\right|\right. \\
&+\left|h \mathrm{HA}\left(\tilde{u}_{h}\right)-h \mathrm{HA}\left(\tilde{u}_{h}^{\prime}\right)\right|+\left|h \mathrm{HA}\left(\tilde{v}_{h}\right)-h \mathrm{HA}\left(\tilde{v}_{h}^{\prime}\right)\right| \\
&\left.+d_{H}\left(h^{2}\left[\tilde{u}_{h}\right]^{h}, h^{2}\left[\tilde{u}_{h}^{\prime}\right]^{h}\right)+d_{H}\left(h^{2}\left[\tilde{v}_{h}\right]^{h}, h^{2}\left[\tilde{v}_{h}^{\prime}\right]^{h}\right)\right\}, \\
&= \frac{1}{2}\left\{\left|h \mathrm{HV}\left(\tilde{u}_{h}\right)-h \mathrm{HV}\left(\tilde{u}_{h}^{\prime}\right)\right|+\left|h \mathrm{HA}\left(\tilde{u}_{h}\right)-h \mathrm{HA}\left(\tilde{u}_{h}^{\prime}\right)\right|\right. \\
&\left.+d_{H}\left(h^{2}\left[\tilde{u}_{h}\right]^{h}, h^{2}\left[\tilde{u}_{h}^{\prime}\right]^{h}\right)\right\}+\frac{1}{2}\left\{\left|h \mathrm{HV}\left(\tilde{v}_{h}\right)-h \mathrm{HV}\left(\tilde{v}_{h}^{\prime}\right)\right|\right. \\
&\left.+\left|h \mathrm{HA}\left(\tilde{v}_{h}\right)-h \mathrm{HA}\left(\tilde{v}_{h}^{\prime}\right)\right|+d_{H}\left(h^{2}\left[\tilde{v}_{h}\right]^{h}, h^{2}\left[\tilde{v}_{h}^{\prime}\right]^{h}\right)\right\}, \\
&= \operatorname{HSD}\left(\tilde{u}_{h}, \tilde{u}_{h}^{\prime}\right)+\operatorname{HSD}\left(\tilde{v}_{h}, \tilde{v}_{h}^{\prime}\right) .
\end{aligned}
$$

In definition of HSD, two source functions $s_{1}$ and $s_{2}$ are defined over $\left[0, h_{1}\right]$ and $\left[0, h_{2}\right]$, respectively. If $s_{1}$ over $\left[0, h_{1}\right]$ is equivalent to $s_{2}$ over $\left[0, h_{2}\right],\left(s_{1} \approx s_{2}\right)$, then we denote this distance by $\mathrm{HSD}_{s}$ with respect to the source function defined over $[0,1]$.

Example 11 In Fig. 1, functions $s_{1}(\alpha)=\frac{1}{2} s(2 \alpha)$ over $\left[0, \frac{1}{2}\right]$, $s_{2}(\alpha)=\frac{1}{5} s(5 \alpha)$ over $\left[0, \frac{1}{5}\right]$ and $s_{h}(\alpha)=\frac{1}{h} s(h \alpha)$ over $\left[0, \frac{1}{h}\right]$ (where $h \geq 1$ ) are all equivalent to the trivial source function $s(\alpha)=\alpha$ over $[0,1]$. 


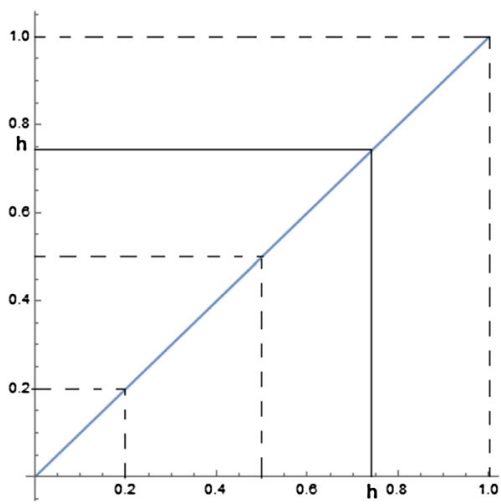

Fig. 1 Some source functions equivalent to the trivial source function

Example 12 In Fig. 2, functions $s_{1}(\alpha)=\frac{1}{2} s(2 \alpha)$ over $\left[0, \frac{1}{2}\right]$ and $s_{2}(\alpha)=\frac{1}{5} s(5 \alpha)$ over $\left[0, \frac{1}{5}\right]$ are both equivalent to a nontrivial source function $s(\alpha)=2 \alpha^{3}-3 \alpha^{2}+2 \alpha$ over [0, 1].

\section{The nearest approximation of a fuzzy semi- number}

In this section, we use HSD to find the nearest approximation of an arbitrary fuzzy semi-number. We start with some definitions and continue with presenting a theorem on the set of all fuzzy semi-numbers with equivalent source functions.

Definition 12 Let $\tilde{u}_{h} \in \mathrm{FS}(\mathbb{R})$ and $h^{*} \in(0,1]$. $\tilde{u}^{*}$ is the nearest approximation to $\tilde{u}_{h}$ out of $F_{h^{*}}(\mathbb{R})$, if and only if

$\operatorname{HSD}\left(\tilde{u}_{h}, \tilde{u}_{h^{*}}^{*}\right)=\min _{\tilde{v}_{h^{*}} \in F_{h^{*}}(\mathbb{R})} \operatorname{HSD}\left(\tilde{u}_{h}, \tilde{v}_{h^{*}}\right)$.

Definition 13 Let $\tilde{u}_{h}$ be an arbitrary fuzzy semi-number. For $h^{*} \in(0,1], \tilde{u}_{h^{*}}^{*}$ is the consistent nearest approximation to $\tilde{u}_{h}$ out of $\operatorname{TFS}(\mathbb{R})$, if and only if

$\operatorname{HSD}\left(\tilde{u}_{h}, \tilde{u}_{h^{*}}^{*}\right)=0$.

Theorem $13 \quad$ Let $\quad \tilde{u}_{h_{u}}, \tilde{v}_{h_{v}} \in T F_{h}(\mathbb{R})$, then $\operatorname{HSD}_{s}\left(\tilde{u}_{h_{u}}, \tilde{v}_{h_{v}}\right)=0$, if and only if $\tilde{u}_{h_{u}}=\tilde{v}_{h_{v}}$.

Proof If $\tilde{u}_{h_{u}}=\tilde{v}_{h_{v}}$, from Theorem 7 we have $\operatorname{HSD}_{s}\left(\tilde{u}_{h_{u}}, \tilde{v}_{h_{v}}\right)=0$.

Conversely, assume $\operatorname{HSD}_{s}\left(\tilde{u}_{h_{u}}, \tilde{v}_{h_{v}}\right)=0$. If $\tilde{u}=0_{h_{u}}^{\circ}$ and $\tilde{v}=0_{h_{v}}^{\circ}$, we have $\left|h_{u}-h_{v}\right|=0$. Thus, $h_{u}=h_{v}$ and $\tilde{u}_{h_{u}}=\tilde{v}_{h_{v}}$. Without any loss of generality let $\tilde{u}_{h_{u}}=$ $\left(a_{u}, b_{u}, c_{u}, d_{u} ; h_{u}\right)$ and $\tilde{v}_{h_{v}}=\left(a_{v}, b_{v}, c_{v}, d_{v} ; h_{v}\right)$ be two trapezoidal fuzzy semi-numbers where $\tilde{u}_{h_{u}} \neq 0_{h_{u}}^{\circ}$ or $\tilde{v}_{h_{v}} \neq 0_{h_{v}}^{\circ}$. If $\operatorname{HSD}_{s}\left(\tilde{u}_{h_{u}}, \tilde{v}_{h_{v}}\right)=0$ then

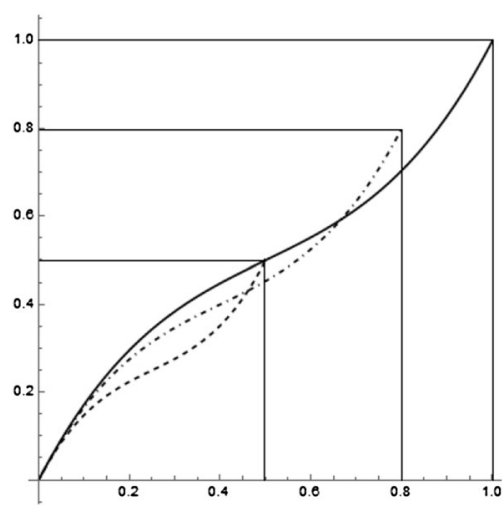

Fig. 2 Some source functions equivalent to a non-trivial source function

$\left\{\begin{array}{cc}(\mathrm{i}) & d_{H}\left(h_{u}^{2}\left[c_{u}, b_{u}\right], h_{v}^{2}\left[c_{v}, b_{v}\right]\right)=0, \\ (\mathrm{ii}) & h_{u} H V\left(\tilde{u}_{h_{u}}\right)=h_{v} H V\left(\tilde{v}_{h_{v}}\right), \\ (\mathrm{iii}) & h_{u} H A\left(\tilde{u}_{h_{u}}\right)=h_{v} H A\left(\tilde{v}_{h_{v}}\right) .\end{array}\right.$

From (i), we have $\max \left\{\left|h_{u}^{2} c_{u}-h_{v}^{2} c_{v}\right|,\left|h_{u}^{2} b_{u}-h_{v}^{2} b_{v}\right|\right\}=0$ and hence $b_{v}=\left(\frac{h_{u}}{h_{v}}\right)^{2} b_{u}$ and $c_{v}=\left(\frac{h_{u}}{h_{v}}\right)^{2} c_{u}$. From (ii) and (iii)

$$
\begin{aligned}
h_{u} H V\left(\tilde{u}_{h_{u}}\right)+h_{u} H A\left(\tilde{u}_{h_{u}}\right)= & 2 h_{u} \int_{0}^{h_{u}} s(\alpha) \bar{u}(\alpha) \mathrm{d} \alpha \\
= & 2 h_{v} \int_{0}^{h_{v}} s(\alpha) \bar{v}(\alpha) \mathrm{d} \alpha \\
= & h_{v} H V\left(\tilde{v}_{h_{v}}\right) \\
& +h_{v} H A\left(\tilde{v}_{h_{v}}\right), h_{u} H V\left(\tilde{u}_{h_{u}}\right) \\
& -h_{u} H A\left(\tilde{u}_{h_{u}}\right) \\
= & 2 h_{u} \int_{0}^{h_{u}} s(\alpha) \underline{u}(\alpha) \mathrm{d} \alpha \\
= & 2 h_{v} \int_{0}^{h_{v}} s(\alpha) \underline{v}(\alpha) \mathrm{d} \alpha \\
= & h_{v} H V\left(\tilde{v}_{h_{v}}\right)-h_{v} H A\left(\tilde{v}_{h_{v}}\right),
\end{aligned}
$$

and hence

$\left\{\begin{array}{l}\frac{d_{u} h_{u}^{3}}{2}-\left(d_{u}-c_{u}\right) I_{s, h_{u}}=\frac{d_{v} h_{v}^{3}}{2}-\left(d_{v}-c_{v}\right) I_{s, h_{v}}, \\ \frac{a_{u} h_{u}^{3}}{2}+\left(b_{u}-a_{u}\right) I_{s, h_{u}}=\frac{a_{v} h_{v}^{3}}{2}+\left(b_{v}-a_{v}\right) I_{s, h_{v}} .\end{array}\right.$

which are equivalent to the following relations:

$\left\{\begin{array}{l}d_{u}\left(h_{u}^{3}-2 I_{s, h_{u}}\right)+2 c_{u} I_{s, h_{u}}=d_{v}\left(h_{v}^{3}-2 I_{s, h_{v}}\right)+2 c_{v} I_{s, h_{v}}, \\ a_{u}\left(h_{u}^{3}-2 I_{s, h_{u}}\right)+2 b_{u} I_{s, h_{u}}=a_{v}\left(h_{v}^{3}-2 I_{s, h_{v}}\right)+2 b_{v} I_{s, h_{v}} .\end{array}\right.$

Since $b_{v}=\left(\frac{h_{u}}{h_{v}}\right)^{2} b_{u}$ and $c_{v}=\left(\frac{h_{u}}{h_{v}}\right)^{2} c_{u}$, we have: 


$$
\left\{\begin{array}{l}
a_{v}=\frac{h_{u}^{3}-2 I_{s, h_{u}}}{h_{v}^{3}-2 I_{s, h_{v}}} a_{u}+\frac{2 h_{v}^{2} I_{s, h_{u}}-2 h_{u}^{2} I_{s, h_{v}}}{h_{v}^{5}-2 h_{v}^{2} I_{s, h_{v}}} b_{u}, \\
d_{v}=\frac{h_{u}^{3}-2 I_{s, h_{u}}}{h_{v}^{3}-2 I_{s, h_{v}}} d_{u}+\frac{2 h_{v}^{2} I_{s, h_{u}}-2 h_{u}^{2} I_{s, h_{v}}}{h_{v}^{5}-2 h_{v}^{2} I_{s, h_{v}}} c_{u} .
\end{array}\right.
$$

By assumption, we have $h_{u}=h_{v}$, it implies that $d_{v}=d_{u}$ and $a_{v}=a_{u}$; therefore, we have $\tilde{u}_{h_{u}}=\tilde{v}_{h_{v}}$.

From Theorem 7, Corollaries 4.2 and 4.3 can be immediately derived.

Corollary $14 \mathrm{HSD}_{s}$, is a metric on $\mathrm{TF}_{h}(\mathbb{R})$, for a fixed height $h$.

Proof By Theorems 7 and 13 the proof is clear.

Corollary 15 Let $\tilde{u}_{h_{u}} \in T F_{h_{u}}(\mathbb{R}), \tilde{v}_{h_{v}} \in T F_{h_{v}}(\mathbb{R})$ and $s_{h_{u}}$ be equivalent to $s_{h_{v}}$. If $\operatorname{HSD}_{s}\left(\tilde{u}_{h_{u}}, \tilde{v}_{h_{v}}\right)=0$, then

$$
\left\{\begin{array}{c}
a_{v}=\left(\frac{h_{u}}{h_{v}}\right)^{3} a_{u}+\frac{2 h_{u}^{2}\left(h_{u}-h_{v}\right) I_{s, h_{u}}}{h_{v}^{3}\left(h_{u}^{3}-2 I_{s, h_{u}}\right)} b_{u}, \\
b_{v}=\left(\frac{h_{u}}{h_{v}}\right)^{2} b_{u}, \\
c_{v}=\left(\frac{h_{u}}{h_{v}}\right)^{2} c_{u}, \\
d_{v}=\left(\frac{h_{u}}{h_{v}}\right)^{3} d_{u}+\frac{2 h_{u}^{2}\left(h_{u}-h_{v}\right) I_{s, h_{u}}}{h_{v}^{3}\left(h_{u}^{3}-2 I_{s, h_{u}}\right)} c_{u} .
\end{array}\right.
$$

Proof By Theorem 7 and Lemma 5 the proof is clear.

For an arbitrary fuzzy semi-number $\tilde{u}_{h_{u}} \in T F_{h_{u}}(\mathbb{R})$ with height $h_{u}$, the consistent nearest approximation $\tilde{v}_{h_{v}}=$ $\left(a_{v}, b_{v}, c_{v}, d_{v} ; h_{v}\right)$ can be found by applying equation (18) for an arbitrary height $h_{v}$

$a_{v}=\frac{h_{u}^{3}-2 I_{s, h_{u}}}{h_{v}^{3}-2 I_{s, h_{v}}} a_{u}+\frac{2 h_{v}^{2} I_{s, h_{u}}-2 h_{u}^{2} I_{s, h_{v}}}{h_{v}^{5}-2 h_{v}^{2} I_{s, h_{v}}} b_{u}$,

$b_{v}=\left(\frac{h_{u}}{h_{v}}\right)^{2} b_{u}$

$c_{v}=\left(\frac{h_{u}}{h_{v}}\right)^{2} c_{u}$

$d_{v}=\frac{h_{u}^{3}-2 I_{s, h_{u}}}{h_{v}^{3}-2 I_{s, h_{v}}} d_{u}+\frac{2 h_{v}^{2} I_{s, h_{u}}-2 h_{u}^{2} I_{s, h_{v}}}{h_{v}^{5}-2 h_{v}^{2} I_{s, h_{v}}} c_{u}$.

Since the approximation depends on the height of known trapezoidal fuzzy semi-number, the trapezoidal approximation may not exist for a specific height. Example 16 explains this case.

Example 16 Let $\tilde{u}=\left(1,2,3,4 ; \frac{1}{2}\right)$ and $s(r)=r$. The consistent nearest approximation of $\tilde{u}$ for height $\frac{1}{3}$, is $\tilde{v}=\left(\frac{63}{8}, \frac{9}{2}, \frac{27}{4}, \frac{81}{4} ; \frac{1}{3}\right)$. Since $a_{v} \geq b_{v}, \tilde{v}$ is not a trapezoidal fuzzy semi-number.
Therefore, in the following subsections, a range is specified for $h$ associated with a non-negative or nonpositive trapezoidal fuzzy semi-number. This range must satisfy some constraints which will be explained in the following subsections.

\section{A constraint on the nearest approximation' height of a non-negative fuzzy semi-number}

Let $\tilde{u}=\left(a_{u}, b_{u}, c_{u}, d_{u} ; h_{u}\right)$ be an arbitrary non-negative trapezoidal fuzzy semi-number, for the consistent nearest approximation via $\mathrm{HSD}_{s}$, with height $h_{v}$, where $\tilde{v}=\left(a_{v}, b_{v}, c_{v}, d_{v} ; h_{v}\right) \in T F_{h_{v}}(\mathbb{R})$, the Lemmas 17 and 18 can be derived.

Lemma 17 If $h_{v} \geq h_{u}$, the H-core of the consistent nearest approximated non-negative trapezoidal fuzzy seminumber, $\tilde{v}_{h_{v}}$, tends to the left side and similarly if $h_{v} \leq h_{u}$, the H-core of the consistent nearest approximated nonnegative trapezoidal fuzzy semi-number, $\tilde{v}_{h_{v}}$, tends to the right side.

Proof When $h_{v} \geq h_{u}$, from (20) and (21) we will have $b_{v} \leq b_{u}$ and $c_{v} \leq c_{u}$, when $h_{v} \leq h_{u}$, from (20) and (21) we will have $b_{v} \geq b_{u}$ and $c_{v} \geq c_{u}$, since $a_{u} \geq b_{u} \geq c_{u} \geq d_{u} \geq 0$, the proof of the lemma is complete.

Lemma 18 Let $\tilde{u}$ be a non-negative trapezoidal fuzzy semi-number with fixed height $h_{u}$. For each $h_{v} \leq h_{u}$ and the source function $s_{h_{v}}$ which is equivalent to $s_{h_{u}}$, we have $c_{v} \leq d_{v}$, similarly for each $h_{v} \geq h_{u}$ we have $a_{v} \leq b_{v}$.

Proof Let $I_{u}=I_{s_{h_{u}}, h_{u}}$ and $I_{v}=I_{s_{h_{v}}, h_{v}}$. Since $s_{h_{u}} \approx s_{h_{v}}$, with respect to Lemma 5 we have

$I_{v}=\left(\frac{h_{v}}{h_{u}}\right)^{3} I_{u}$.

From Eqs. (21) and (22) we can write as follows:

$d_{v}-c_{v}=\frac{h_{u}^{3}-2 I_{u}}{h_{v}^{3}-2 I_{v}} d_{u}+\frac{2 h_{v}^{2} I_{u}-2 h_{u}^{2} I_{v}}{h_{v}^{5}-2 h_{v}^{2} I_{v}} c_{u}-\left(\frac{h_{u}}{h_{v}}\right)^{2} c_{u}$,

then by Eq. (23),

$$
\begin{aligned}
d_{v}-c_{v} & =\left(\frac{h_{u}}{h_{v}}\right)^{3}\left(\frac{d_{u} h_{u}^{3}-c_{u} h_{u}^{2} h_{v}+2\left(c_{u}-d_{u}\right) I_{u}}{h_{u}^{3}-2 I_{u}}\right), \\
& =\left(\frac{h_{u}}{h_{v}}\right)^{3}\left(d_{u}-c_{u}\right)\left(\frac{h_{u}^{2}\left(\frac{d_{u} h_{u}-c_{u} h_{v}}{d_{u}-c_{u}}\right)-2 I_{u}}{h_{u}^{3}-2 I_{u}}\right),
\end{aligned}
$$

since $\quad d_{u} \geq c_{u} \geq 0 \quad$ and $\quad h_{u} \geq h_{v}$, we have $d_{u} h_{u}-$ $c_{u} h_{v} \geq d_{u} h_{u}-c_{u} h_{u}$ and from that we have $\left(\frac{d_{u} h_{u}-c_{u} h_{v}}{d_{u}-c_{u}}\right) \geq h_{u}$. By multiply $h_{u}^{2}$ to both sides and subtract $2 I_{u}$ from both side we'll get $h_{u}^{2}\left(\frac{d_{u} h_{v}-c_{u} h_{u}}{d_{u}-c_{u}}\right)-2 I_{u} \geq h_{u}^{3}-2 I_{u}$. By Lemma 6 and $d_{u}-c_{u} \geq 0$, 
$h_{u}^{2} \frac{\left(d_{u} h_{v}-c_{u} h_{u}\right)}{\left(d_{u}-c_{u}\right)}-2 I_{u} \geq h_{u}^{3}-2 I_{u}>0$,

hence $d_{v}-c_{v} \geq 0$.

Similarly for $h_{v} \geq h_{u}$ from Eqs. (20), (19), (23) and $b_{u}-a_{u} \geq 0$, we have:

$b_{v}-a_{v}=\left(\frac{h_{u}}{h_{v}}\right)^{3}\left(b_{u}-a_{u}\right)\left(\frac{h_{u}^{2}\left(\frac{b_{u} h_{v}-a_{u} h_{u}}{b_{u}-a_{u}}\right)-2 I_{u}}{h_{u}^{3}-2 I_{u}}\right) \geq 0$.

Theorem 19 Let s be a source function, for an arbitrary known non-negative trapezoidal fuzzy semi-number $\tilde{u}=$ $\left(a_{u}, b_{u}, c_{u}, d_{u} ; h_{u}\right)$ with height $h_{u}$. The consistent nearest approximation via $\mathrm{HSD}_{s}$ is a trapezoidal fuzzy semi-number with height $h_{v}$, if and only if $h_{v}$ satisfies one of the following conditions:

(i) If $h_{v} \leq h_{u}$, then

$$
h_{v} \geq \frac{a_{u} h_{u}^{3}-2 a_{u} I_{s, h_{u}}+2 b_{u} I_{s, h_{u}}}{b_{u} h_{u}^{2}},
$$

(ii) If $h_{v} \geq h_{u}$, then

$$
h_{v} \leq \frac{d_{u} h_{u}^{3}-2 d_{u} I_{s, h_{u}}+2 c_{u} I_{s, h_{u}}}{c_{u} h_{u}^{2}} .
$$

Proof Let $I_{u}=I_{s_{h_{u}}, h_{u}}, I_{v}=I_{s_{h_{v}}}, h_{v}$ and $\tilde{v}=\left(a_{v}, b_{v}, c_{v}, d_{v} ; h_{v}\right)$ be the consistent nearest approximation of $\tilde{u}_{h_{u}}$. $\tilde{v}_{h_{v}}$ is a trapezoidal fuzzy semi-number if $a_{v} \leq b_{v} \leq c_{v} \leq d_{v}$. From Eqs. (20) and (21) it is clear that $b_{v} \leq c_{v}$.

(i) From Lemma 17 and 18, whenever $h_{v} \leq h_{u}$, it is sufficient that $a_{v} \leq b_{v}$ holds, therefore, by Eqs. (20) and (19) we write as follows:

$$
\begin{aligned}
b_{v}-a_{v}= & \left(\frac{h_{u}}{h_{v}}\right)^{2} b_{u}-\frac{h_{u}^{3}-2 I_{u}}{h_{v}^{3}-2 I_{v}} a_{u} \\
& -\frac{2 h_{v}^{2} I_{u}-2 h_{u}^{2} I_{v}}{h_{v}^{5}-2 h_{v}^{2} I_{v}} b_{u}, \\
= & \left(\frac{h_{u}}{h_{v}}\right)^{3}\left(\frac{-a_{u} h_{u}^{3}+b_{u} h_{u}^{2} h_{v}+2 a_{u} I_{u}-2 b_{u} I_{u}}{h_{u}^{3}-2 I_{u}}\right),
\end{aligned}
$$

From Lemma $6, b_{v} \geq a_{v}$ implies that

$-a_{u} h_{u}^{3}+b_{u} h_{u}^{2} h_{v}+2 a_{u} I_{u}-2 b_{u} I_{u} \geq 0$,

since $b_{u} \geq a_{u} \geq 0$ hence

$h_{v} \geq \frac{a_{u} h_{u}^{3}-2 a_{u} I_{u}+2 b_{u} I_{u}}{b_{u} h_{u}^{2}}$.

(ii) From Lemmas 17 and 18 , whenever $h_{v} \geq h_{u}$ it is sufficient that $c_{v} \leq d_{v}$ holds; therefore, by Eqs. (21) and (22) we write as follows: $d_{v}-c_{v}=\left(\frac{h_{u}}{h_{v}}\right)^{3} \frac{d_{u} h_{u}^{3}-c_{u} h_{u}^{2} h_{v}-2 d_{u} I_{u}+2 c_{u} I_{u}}{h_{u}^{3}-2 I_{u}}$,

From Lemma $6, d_{v} \geq c_{v}$ implies that

$d_{u} h_{u}^{3}-c_{u} h_{u}^{2} h_{v}-2 d_{u} I_{u}+2 c_{u} I_{u} \geq 0$

since $d_{u} \geq c_{u} \geq 0$, then

$h_{v} \leq \frac{d_{u} h_{u}^{3}-2 d_{u} I_{u}+2 c_{u} I_{u}}{c_{u} h_{u}^{2}}$.

Now, the Corollaries 20 and 21 can be derived from Theorem 19.

Corollary 20 Let $\tilde{u}=\left(a_{u}, b_{u}, c_{u}, d_{u} ; h\right)$ be an arbitrary non-negative fuzzy semi-number with height $h$. When $1 \leq \frac{d_{u} h^{3}-2 d_{u} I_{s, h}+2 c_{u} I_{s, h}}{c_{u} h^{2}}$, the consistent nearest trapezoidal fuzzy number of $\tilde{u}_{h}$ is $\tilde{v}=\left(a_{v}, b_{v}, c_{v}, d_{v}\right)$, where

$$
\begin{aligned}
& a_{v}=\frac{h^{3}-2 I_{s, h}}{1-2 I_{s, 1}} a_{u}+\frac{2 I_{s, h}-2 h^{2} I_{s, 1}}{1-2 I_{s, 1}} b_{u}, \\
& b_{v}=h^{2} b_{u}, \\
& c_{v}=h^{2} c_{u}, \\
& d_{v}=\frac{h^{3}-2 I_{s, h}}{1-2 I_{s, 1}} d_{u}+\frac{2 I_{s, h}-2 h^{2} I_{s, 1}}{1-2 I_{s, 1}} c_{u} .
\end{aligned}
$$

Proof Straightforward.

Corollary 21 Let $\tilde{u}=\left(a_{u}, b_{u}, c_{u}, d_{u}\right)$ be an arbitrary nonnegative trapezoidal fuzzy number. The consistent nearest fuzzy semi-number of $\tilde{u}_{h}$ with height $h$, for $h \geq \frac{a_{u}-2 a_{u} I_{s, 1}+2 b_{u} I_{s, 1}}{b_{u}}$ is $\tilde{v}=\left(a_{v}, b_{v}, c_{v}, d_{v} ; h\right)$, where

$a_{v}=\frac{1-2 I_{s, 1}}{h^{3}-2 I_{s, h}} a_{u}+\frac{2 h^{2} I_{s 1}-2 I_{s, h}}{h^{5}-2 h^{2} I_{s, h}} b_{u}$,

$b_{v}=\left(\frac{1}{h}\right)^{2} b_{u}$,

$c_{v}=\left(\frac{1}{h}\right)^{2} c_{u}$,

$d_{v}=\frac{1-2 I_{s, 1}}{h^{3}-2 I_{s, h}} d_{u}+\frac{2 h^{2} I_{s, 1}-2 I_{s, h}}{h^{5}-2 h^{2} I_{s, h}} c_{u}$.

Proof Straightforward.

\section{A constraint on the nearest approximation' height of a non-positive fuzzy semi-number}

Let $\tilde{u}=\left(a_{u}, b_{u}, c_{u}, d_{u} ; h_{u}\right)$ be an arbitrary known non-positive trapezoidal fuzzy semi-number, for the consistent nearest approximation via $\mathrm{HSD}_{s}$, with height $h_{v}$, where $\tilde{v}=$ $\left(a_{v}, b_{v}, c_{v}, d_{v} ; h_{v}\right) \in F_{h_{v}}(\mathbb{R})$, Lemmas 22 and 23 are obtained. 
Lemma 22 If $h_{v} \geq h_{u}$ the H-core of the consistent nearest approximated non-positive trapezoidal fuzzy semi-number, $\tilde{v}_{h_{v}}$, tends to the right side and similarly if $h_{v} \leq h_{u}$ the H-core of the consistent nearest approximated non-positive trapezoidal fuzzy semi-number, $\tilde{v}_{h_{v}}$, tends to the left side.

Lemma 23 Let $\tilde{u}_{h_{u}} \in T F_{h_{u}}(\mathbb{R})$ and $\tilde{u}_{h_{u}}$ be non-positive for a fixed $h_{u}$. For each $h_{v} \leq h_{u}$ and the source function $s_{h_{v}}$ which is equivalent to $s_{h_{u}}$, we have $a_{v} \leq b_{v}$, similarly for each $h_{v} \geq h_{u}$ we have $c_{v} \leq d_{v}$.

Theorem 24 Let $s$ be a source function, for an arbitrary known non-positive trapezoidal fuzzy semi-number $\tilde{u}=$ $\left(a_{u}, b_{u}, c_{u}, d_{u} ; h_{u}\right)$ with height $h_{u}$. The consistent nearest approximation via $\mathrm{HSD}_{s}$ is a trapezoidal fuzzy semi-number with height $h_{v}$, if and only if $h_{v}$ satisfies one of the following conditions:

(i) If $h_{v} \leq h_{u}$, then

$$
h_{v} \geq \frac{d_{u} h_{u}^{3}-2 d_{u} I_{u}+2 c_{u} I_{u}}{c_{u} h_{u}^{2}},
$$

(ii) If $h_{v} \geq h_{u}$, then

$$
h_{v} \leq \frac{a_{u} h_{u}^{3}-2 a_{u} I_{u}+2 b_{u} I_{u}}{b_{u} h_{u}^{2}} .
$$

Corollary 25 Let $\tilde{u}=\left(a_{u}, b_{u}, c_{u}, d_{u} ; h\right)$ be an arbitrary non-positive fuzzy semi-number with height $h$. When $1 \leq \frac{a_{u} h^{3}-2 a_{u} I_{u}+2 b_{u} I_{u}}{b_{u} h^{2}}$, the consistent nearest trapezoidal fuzzy number of $\tilde{u}_{h}$ is $\tilde{v}=\left(a_{v}, b_{v}, c_{v}, d_{v}\right)$, where

$a_{v}=\frac{h^{3}-2 I_{s, h}}{1-2 I_{s, 1}} a_{u}+\frac{2 I_{s, h}-2 h^{2} I_{s, 1}}{1-2 I_{s, 1}} b_{u}$,

$b_{v}=h^{2} b_{u}$,

$c_{v}=h^{2} c_{u}$,

$d_{v}=\frac{h^{3}-2 I_{s, h}}{1-2 I_{s, 1}} d_{u}+\frac{2 I_{s, h}-2 h^{2} I_{s, 1}}{1-2 I_{s, 1}} c_{u}$.

Corollary 26 Let $\tilde{u}=\left(a_{u}, b_{u}, c_{u}, d_{u}\right)$ be an arbitrary nonpositive trapezoidal fuzzy number. The consistent nearest fuzzy semi-number of $\tilde{u}$ with height $h$ for $h \geq \frac{a_{u}-2 a_{u} I_{s, 1}+2 b_{u} I_{s, 1}}{b_{u}}$, is $\tilde{v}=\left(a_{v}, b_{v}, c_{v}, d_{v} ; h\right)$, where

$a_{v}=\frac{1-2 I_{s, 1}}{h^{3}-2 I_{s, h}} a_{u}+\frac{2 h^{2} I_{s 1}-2 I_{s, h}}{h^{5}-2 h^{2} I_{s, h}} b_{u}$,

$b_{v}=\left(\frac{1}{h}\right)^{2} b_{u}$,

$c_{v}=\left(\frac{1}{h}\right)^{2} c_{u}$

$d_{v}=\frac{1-2 I_{s, 1}}{h^{3}-2 I_{s, h}} d_{u}+\frac{2 h^{2} I_{s, 1}-2 I_{s, h}}{h^{5}-2 h^{2} I_{s, h}} c_{u}$.
The proofs of the lemmas, theorem and corollaries in this subsection are the same as the previous subsection ("A constraint on the nearest approximation' height ofa nonnegative fuzzy semi-number"). Alternatively, these proofs can be derived, assuming $\tilde{u}^{+}=-\tilde{u}$ and $\tilde{v}^{+}=-\tilde{v}$.

\section{Numerical examples}

In this section, we present some numerical examples using the proposed method during paper. The simplest source function is identity function and because of computational complexity we use $s(r)=r$ in our examples.

Example 27 Let $\tilde{u}=\left(1,2,3,4 ; \frac{1}{2}\right)$ and $s(r)=r$. The consistent nearest trapezoidal fuzzy semi-number of $\tilde{u}$ with height $\frac{7}{13}$, is $\tilde{v}=\left(\frac{1521}{2744}, \frac{169}{98}, \frac{507}{196}, \frac{3887}{1372} ; \frac{7}{13}\right)$. In Fig. 3, $\tilde{u}$ and $\tilde{v}$ are shown by solid and dashed lines, respectively.

The nearest trapezoidal fuzzy semi-number of $\tilde{u}$ with height $\frac{6}{13}$, is $\tilde{w}=\left(\frac{2873}{1728}, \frac{169}{72}, \frac{169}{48}, \frac{4901}{864} ; \frac{6}{13}\right)$. In Fig. $4, \tilde{u}$ and $\tilde{w}$ are shown by solid and dashed lines, respectively.

If $h_{v} \geq \frac{1}{2}$, then from Theorem 19 we should have $h_{v} \leq \frac{5}{9}$ and if $h_{v} \leq \frac{1}{2}$, then from Theorem 19 we should have $h_{v} \geq \frac{5}{12}$. Since $\tilde{u}$ is positive and $h_{v} \geq h_{u}, \tilde{v}$ tend to left side as it can be seen in Fig. 3 regarding Lemma 17, it was predictable and because $h_{w} \leq h_{u}, \tilde{w}$ tend to right side as it can be seen in Fig. 4 according to Lemma 17 it could be foretold.

Example 28 Let $\tilde{u}=\left(-5,-3,-2,-1, \frac{3}{4}\right)$ and $s(r)=r$. The consistent nearest fuzzy semi-number of $\tilde{u}$ with height $\frac{7}{8}$, is $\tilde{v}=\left(-\frac{864}{343},-\frac{108}{49},-\frac{72}{49},-\frac{72}{343}, \frac{7}{8}\right)$. In Fig. $4, \tilde{u}$ and $\tilde{v}$ are shown by solid and dashed lines, respectively.

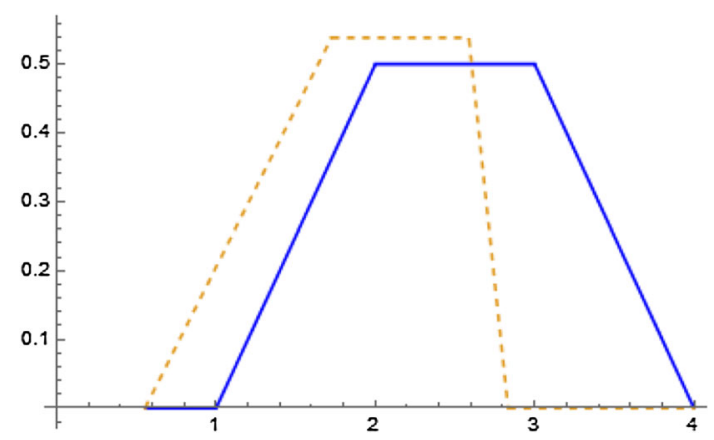

Fig. 3 The nearest trapezoidal fuzzy semi-number with height $\frac{7}{13}$ 


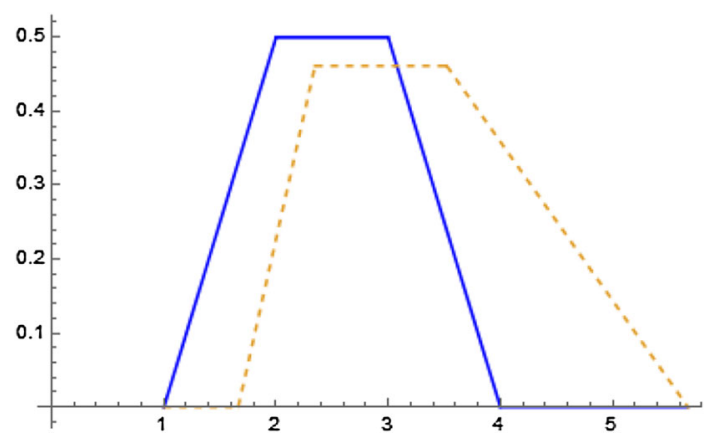

Fig. 4 The nearest trapezoidal fuzzy semi-number with height $\frac{6}{13}$

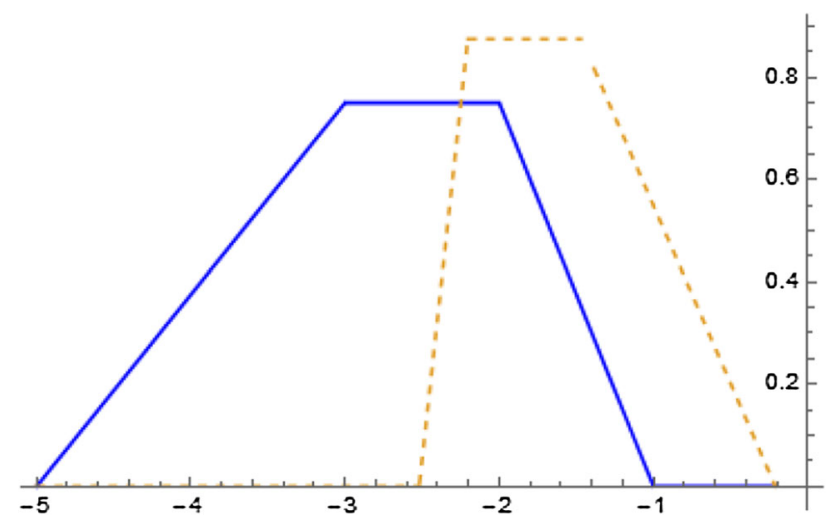

Fig. 5 The nearest fuzzy semi-number with height $\frac{7}{8}$

The nearest fuzzy semi-number of $\tilde{u}$ with height $\frac{2}{3}$, is $\tilde{w}=\left(-\frac{4131}{512},-\frac{243}{64},-\frac{81}{32},-\frac{1053}{512}, \frac{2}{3}\right)$. In Fig. 5, $\tilde{u}$ and $\tilde{w}$ are shown by solid and dashed lines, respectively.

Example 29 Let $\tilde{u}=\left(1,2,3,5 ; \frac{1}{2}\right)$ and $s(r)=r$. The consistent nearest trapezoidal fuzzy semi-number of $\tilde{u}$ with height $\frac{11}{21}, \frac{4}{7}, \frac{10}{22}$ and $\frac{21}{44}$ are as follows:

$$
\begin{aligned}
\tilde{m} & =\left(\frac{7497}{10648}, \frac{441}{242}, \frac{1323}{484}, \frac{3969}{968} ; \frac{11}{21}\right), \tilde{n} \\
& =\left(\frac{147}{512}, \frac{49}{32}, \frac{147}{64}, \frac{1421}{512} ; \frac{4}{7}\right), \tilde{v} \\
& =\left(\frac{363}{200}, \frac{121}{50}, \frac{363}{100}, \frac{7381}{1000} ; \frac{10}{22}\right), \tilde{w} \\
& =\left(\frac{12584}{9261}, \frac{968}{441}, \frac{484}{147}, \frac{56144}{9261} ; \frac{21}{44}\right) .
\end{aligned}
$$

In Fig. 5, $\tilde{u}, \tilde{m}, \tilde{n}, \tilde{v}$ and $\tilde{w}$ are shown .

If $h_{v} \geq \frac{1}{2}$, then from Theorem 19 we should have $h_{v} \leq \frac{3}{5}$ and if $h_{v} \leq \frac{1}{2}$, then from Theorem 19 we should have $h_{v} \geq \frac{9}{22}$. Hence, when $h_{u}=\frac{1}{2}$, given height should belong to $\left[\frac{9}{22}, \frac{3}{5}\right]$; otherwise, the nearest approximation of $\tilde{u}$ will not be a trapezoidal fuzzy semi-number. Since $\tilde{u}$ is a positive semi-number by Lemma 17 as it can be seen in Fig. 5

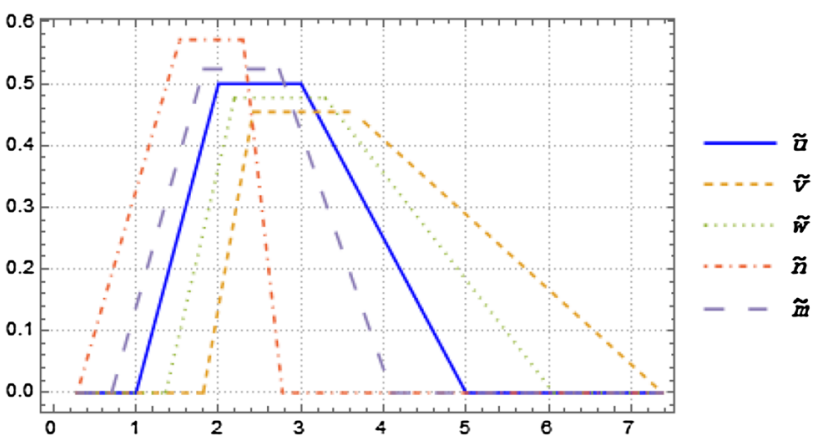

Fig. 6 The nearest trapezoidal fuzzy semi-number with height $\frac{11}{21}, \frac{4}{7}, \frac{10}{22}$ and $\frac{21}{44}$

whenever the given height increases the nearest approximation of $\tilde{u}$ tend to left and whenever the given height decreases the nearest approximation of $\tilde{u}$ tend to right. As it can be seen in Fig. 5, according to Lemma 18 when the given height increases we do not have to worry about left spread and when the given height decreases we do not have to worry about right spread.

Example 30 Let $\tilde{u}=\left(0,2,4,5 ; \frac{1}{2}\right)$ and $\tilde{v}=\left(1,2,3,4 ; \frac{3}{4}\right)$ as shown in Fig. 6. These can be written as $\tilde{u}(r)=(4 r, 5-$ $2 r)$ and $\tilde{v}(r)=\left(1+\frac{4}{3} r, 4-\frac{4}{3} r\right)$. By using $s(r)=r, H$ value and $H$-ambiguity of them can be found as follows:

$$
\begin{aligned}
& \operatorname{HV}\left(\tilde{u}_{h_{u}}\right)=\frac{17}{24}, \\
& \text { HA }\left(\tilde{u}_{h_{u}}\right)=\frac{3}{8}, \quad \operatorname{HV}\left(\tilde{v}_{h_{v}}\right)=\frac{45}{32}, \\
& \operatorname{HA}\left(\tilde{v}_{h_{v}}\right)=\frac{15}{32} .
\end{aligned}
$$

The Height Source Distance between these fuzzy seminumbers is

$\operatorname{HSD}_{s}\left(\tilde{u}_{h_{u}}, \tilde{v}_{h_{v}}\right)=\frac{149}{192}$.

\section{A medical case study}

Without a doubt, controlling the depth of anesthesia is the main task of an anesthetist during surgery. According to the report in [17], annually, anesthesia is the direct responsible for the death of approximately 34 patients. Also it plays an indirect role in another 281 deaths especially in the elderly. Measuring the depth of anesthesia is inherently not straightforward. Thus, anesthetists solve the matter via measuring some other available factors such as blood pressure, heart rate and so on.

In [12], Cullen showed that there is a plausible correlation between blood pressure and anesthetic dose. In other words, gaining a sufficient depth of anesthesia is feasible through controlling the blood pressure related factors such as the MAP (Mean Arterial Pressure which is measured in 


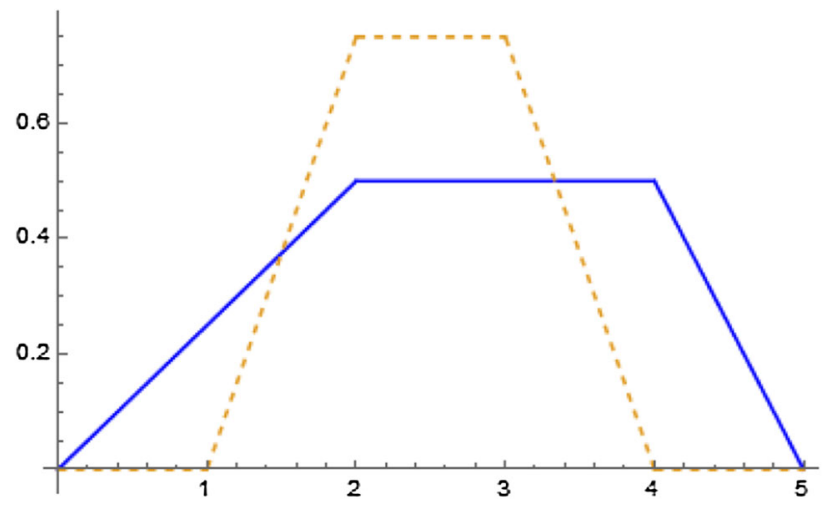

Fig. 7 Two fuzzy semi-numbers $\tilde{u}=\left(0,2,4,5 ; \frac{1}{2}\right)$ and $\tilde{v}=\left(1,2,3,4 ; \frac{3}{4}\right)$

$\mathrm{mmHg}$ ) so that it falls within a predetermined range. This approach has been one of the most prevalent ones in determining the necessary anesthetic dose for decades.

Surgical operations are so risky that most often a small oversight may lead to serious losses. Thus, it is reasonable to automate such tasks as far as possible. In reality, controlling of depth of anesthesia automatically is of highly importance among these tasks, and it releases the surgery staff to pay much more attention to the other activities in the operating room.

There are actually two ways to anesthetize a patient. This is performed either by intravenous injection of drugs or inhaling gasses which are most often a mixture of Isoflurane in oxygen and potentially nitrous oxide. The concentration of Isoflurane used in the process is determined and tuned regarding the type of surgery and the patients psychological condition.

As a case study, in this paper, a fuzzy logic controller is deployed to measure the MAP [21, 22]. This controller simulates the relationship between the inflow concentration of Isoflurane, and the blood pressure. Assume that we are given a fuzzy semi-number which is the average of patient's blood pressure. Due to some disturbances, the blood pressure measurement is an ambiguous process; thus, the given data should be a fuzzy set. Actually, there are different sorts of disturbances such as surgical disturbances to the patient and measurement noise which cause the ambiguity of blood pressure measurement to increase. For this given fuzzy semi-number we find the range of heights which indicates blood pressure variation. Then, we estimate a new fuzzy semi-number with given height related to the new blood pressure to help the anesthetist determine the dose of drugs through the anesthetic process.

The given fuzzy semi-number $\tilde{B}_{h_{B}}=(1,2,3,5 ; 0.67)$, shown in Fig. 7 , represents the uncertainty in each blood pressure measurement. Here, the height of given seminumber, $h_{B}=0.67$, is proportional to blood pressure and its support $[1,5]$ shows the anesthesia duration.

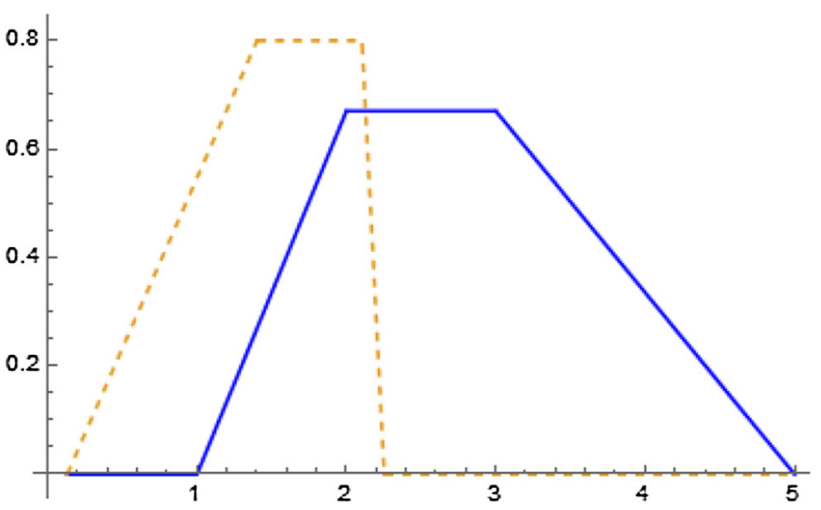

Fig. 8 The solid line shows $\tilde{B}_{h_{B}}$ and the dashed line shows $\tilde{B}_{h_{B^{*}}}^{*}$

To this end, we can estimate the range of height associated with the given fuzzy semi-number with (24) and (25) as follows:

$0.548182 \leqslant h_{\tilde{B}} \leqslant 0.804$

This range shows how the blood pressure varies with the inflow concentration of Isoflurane by applying related defuzzification methods in medicine (Fig. 8).

Moreover, if we want to change blood pressure we approximate a new fuzzy semi-number, $\tilde{B}_{h_{B^{*}}}^{*}$, where $h_{B^{*}}=$ 0.8 is related to this new blood pressure by using (19)-(22) as follows:

$\tilde{B}_{h_{B^{*}}}^{*}=(0.131514,1.40281,2.10422,2.25327 ; 0.8)$

With this approximated fuzzy semi-number we will allow the anesthetist to determine the next drug application for the patient.

\section{Conclusion}

In most of the works on fuzzy sets, the fuzzy sets are convex and normal (the height is one) which are called fuzzy numbers. Approximation of a fuzzy set has been done in several ways. Assigning a single crisp number to a fuzzy set, defining an interval as an approximation of a fuzzy set, defining distance function and solving an optimization problem in order to obtain a trapezoidal fuzzy set as a nearest approximation are among the most well-known ones. All of these methods suffer from some deficiencies such as precision loss. Moreover, none of them have addressed fuzzy sets with heights less than one. Since too many subjects work on non-normal fuzzy sets, in this paper we worked on fuzzy sets in general, without regard to their height. At first, we reviewed the novel concept of fuzzy semi-numbers. Then, we proposed a distance to approximate an arbitrary fuzzy semi-number. Also, as we observed, depending on some predefined conditions, the 
result of approximation can be either a fuzzy number or a fuzzy semi-number. We finished the paper with some numerical examples and presented a critical medicine case study which improved anesthetist decision for prescribing dose of drugs and controlling the depth of anesthesia with our approximation method.

Open Access This article is distributed under the terms of the Creative Commons Attribution 4.0 International License (http://creative commons.org/licenses/by/4.0/), which permits unrestricted use, distribution, and reproduction in any medium, provided you give appropriate credit to the original author(s) and the source, provide a link to the Creative Commons license, and indicate if changes were made.

\section{References}

1. Abbasbandy, S., Asady, B.: The nearest trapezoidal fuzzy number to a fuzzy quantity. Appl. Math. Comput. 156, 381-386 (2004)

2. Abbasbandy, S., Amirfakhrian, M.: The nearest trapezoidal form of a generalized left right fuzzy number. J. Approx. Reason. 43, 166-178 (2006)

3. Abbasbandy, S., Hajjari, T.: A new approach for ranking of trapezoidal fuzzy numbers. Comput. Math. Appl. 57, 413-419 (2009)

4. Abbasbandy, S., Nuraei, R., Ghanbari, M.: Revision of sign distance method for ranking of fuzzy numbers. Iran. J. Fuzzy Syst. 10(4), 101-117 (2013)

5. Amirfakhrian, M.: Properties of parametric form approximation operator of fuzzy numbers. Analele Stiintifice ale Universitatii Ovidius Constanta 18, 23-34 (2010)

6. Anzilli, L., Facchinetti, G., Mastroleo, G.: A parametric approach to evaluate fuzzy quantities. Fuzzy Sets Syst. 250, 110-133 (2014)

7. Ban, A.I., Coroianu, L., Grzegorzewski, P.: Trapezoidal approximation and aggregation. Fuzzy Sets Syst. 177(1), 45-59 (2011)

8. Ban, A.I., Coroianu, L., Khastan, A.: Conditioned weighted LR approximations of fuzzy numbers. Fuzzy Sets Syst. 62, 1-26 (2015)
9. Chalco Cano, Y., Bez-Snchez, A.D., Romn-Flores, H., RojasMedar, M.A.: On the approximation of compact fuzzy sets. Comput. Math. Appl. 61, 412-420 (2011)

10. Chanas, S.: On the interval approximation of a fuzzy number. Fuzzy Sets Syst. 122, 353-356 (2001)

11. Coroianu, L., Gagolewskic, M., Grzegorzewski, P.: Nearest piecewise linear approximation of fuzzy numbers. Fuzzy Sets Syst. 233, 26-51 (2013)

12. Cullen, D.J., et al.: Clinical signs of anesthesia. Anesthesiology 36, 21-36 (1972)

13. Delgado, M., Vila, M.A., Voxman, W.: On a canonical representation of fuzzy numbers. Fuzzy Sets Syst. 93, 125-135 (1998)

14. Grzegorzewski, P.: Nearest interval approximation of a fuzzy number. Fuzzy Sets Syst. 130, 321-330 (2002)

15. Grzegorzewski, P., Mrówka, E.: Trapezoidal approximations of fuzzy numbers. In: Bilgic, T., De Baets, B., Kaynak O. (eds.) Fuzzy Sets and Systems, IFSA 2003, Lecture Notes in Artificial Intelligence, vol. 2715, pp. 237-244. Springer, New York (2003)

16. Grzegorzewski, P., Mrówka, E.: Trapezoidal approximations of fuzzy numbers. Fuzzy Sets Syst. 153, 115-135 (2005)

17. Li, G., Warner, M., Lang, B.H., Huang, L., Sun, L.S.: Epidemiology of anesthesia-related mortality in the United States, 1999-2005. Anesthesiology 110(4), 759-765 (2009)

18. Ma, M., Kandel, A., Friedman, M.: A new approach for defuzzification. Fuzzy Sets Syst. 111, 351-356 (2000)

19. Ma, M., Kandel, A., Friedman, M.: Correction to "A new approach for defuzzification". Fuzzy Sets Syst. 128, 133-134 (2002)

20. Voxman, W.: Some remarks on distance between fuzzy numbers. Fuzzy Sets Syst. 100, 353-365 (1998)

21. Ying, H., Sheppard, L.C.: Regulating mean arterial pressure in postsurgical cardiac patients. IEEE Eng. Med. Biol. Mag. 13(5), 671-677 (1994)

22. Zbinden, A.M., Feigenwinter, P., Petersen-felix, S., Hacisalizade, S.: Arterial pressure control with isoflurane using fuzzy logic. Br. J. Anaesth. 74, 66-72 (1995)

23. Zimmermann, H.J.: Fuzzy Set Theory and Its Applications, 2nd edn. Kluwer Academic, Boston (1991)

\section{Publisher's Note}

Springer Nature remains neutral with regard to jurisdictional claims in published maps and institutional affiliations. 